Contacts:

Zaitseva Lyudmila Yuryevna, Candidate of Medical Sciences, Assistant of the Department of Pediatrics at the KSMU SBEI HPE, MH RF Address: 3 Karl Marx Str., Kursk 305004, Tel.: (4712) 54-92-90, e-mail: luda201235@yandex.ru

Article received: 01.02.2013, Accepted for publication: 28.10.2013

Цель исследования: оценить особенности течения заболевания, формирования сопутствующей патологии, а также состояние ферментных систем желудочно-кишечного тракта у детей с хроническим гастродуоденитом, проживающих на территории Курской магнитной аномалии. Пациенты и методы: проведено комплексное обследование 120 детей с верифицированным диагнозом: “Хронический гастродуоденит" (60 пациентов из г. Курска и 60 из г. Железногорска). Результаты: установлено, что хронические заболевания желудка и двенадцатиперстной кишки у детей Железногорска регистрируют в 2,7 раза чаще, чем в Курске. Обращает на себя внимание ранняя манифестация хронического гастродуоденита у детей Железногорска. Средний возраст дебюта заболевания в Курске равен 6,4 \pm 0,4 года, тогда как в Железногорске он составил 5,0 \pm 0,4 года ( $p \leqslant 0,01)$. В качестве сопутствующей патологии у детей в Железногорске чаще встречались реактивные изменения гепатобилиарной зоны и сочетание хронического гастродуоденита с аллергопатологией. При изучении копрограмм у большинства пациентов из Железногорска выявлено достоверно большее содержание солей жирных кислот в кале. Выводы: указанные изменения могут свидетельствовать о недостаточности ферментативной активности отдельных компонентов пищеварительных соков. Детей с хроническим гастродуоденитом, проживающих на территории Курской магнитной аномалии, следует выделить в особую группу пациентов. Это даст основания для рациональной коррекции стандартных схем их лечения с учетом обнаруженных особенностей.

Ключевые слова: дети, хронический гастродуоденит, аномальное геомагнитное поле.

(Вопросы современной педиатрии. 2013; 12 (5): 90-93)

\section{ВВЕДЕНИЕ}

Заболевания желудка и двенадцатиперстной кишки являются наиболее распространенными из всех болезней органов пищеварения у детей и составляют
58-65\% в общей структуре детской гастроэнтерологической патологии [1-4]. На долю хронического гастродуоденита (ХГД) приходится почти 45\% заболеваемости детей младшего, $73 \%$ - среднего и 65\% - старшего

L.Yu. Zaitseva, P.V. Kalutskii

Kursk State Medical University, Russian Federation

\title{
Special Characteristics of Chronic Gastroduodenal Disorders Development in Children from a Region with Abnormal Intensity of Geomagnetic Field
}

Aim: to assess special characteristics of disease course, development of concomitant diseases and enzymatic status of gastro-intestinal tract in children with chronic gastroduodenitis, living over the territory of Kursk magnetic anomaly. Patients and methods: 120 children with confirmed diagnosis of chronic gastroduodenitis (among them 60 patients from Kursk and 60 patients from Zheleznogorsk) were performed complex clinical examination. Results: the prevalence of gastroduodenal disorders were 2,7 times higher among children from Zheleznogorsk, than among children from Kursk. Early beginning of chronic gastroduodenitis in children of Zheleznogorsk must be mentioned. The mean age of the onset of the disease in Kursk is $6,4 \pm 0,4$ years, while in Zheleznogorsk $-5,0 \pm 0,4$ years $(p<0,01)$. The most common concomitant diseases in Zheleznogorsk children were reactive changes of hepatobiliary system and combination of chronic gastroduodenitis with allergic pathology. On coprological test the majority of Zheleznogorsk patients had significantly higher content of fatty acids in faeces. Conclusions: the above-mentioned changes can be evidence of insufficient enzymatic activity of certain components of digestive juices. Children with chronic gastroduodenitis living over the territory of Kursk magnetic anomaly should be isolated into a separate group of patients. It will allow rational correction of standard schemes of treatment with accordance to established characteristics.

Key words: children, chronic gastroduodenitis, abnormal geomagnetic field. (Voprosy sovremennoi pediatrii - Current Pediatrics. 2013; 12 (5): 90-93) 
школьного возраста $[5,6]$. При этом снижение относительной частоты ХГД с возрастом происходит за счет увеличения доли язвенной болезни $[7,8]$. В связи с этим рассмотрение этой патологии с позиции нерешенных проблем представляется весьма актуальным. За последние 20 лет отмечено значительное увеличение числа случаев заболеваний желудочно-кишечного тракта у детей в Российской Федерации. При этом имеет место неконтролируемое повышение частоты встречаемости гастродуоденитов $[9,10]$. Только у $10-15 \%$ детей встречается изолированное поражение желудка или двенадцатиперстной кишки (гастрит или дуоденит), в остальных 85-90\% случаев оно носит сочетанный характер, что указывает на общность механизмов их развития [11].

Широкая распространенность заболеваний желудочно-кишечного тракта приводит к формированию стойких нарушений процессов роста и развития детей, снижению качества их жизни, создает предпосылки к развитию мультисистемной патологии. Незрелость тонких механизмов иммунной и нервной регуляции делает организм ребенка более уязвимым к воздействиям большого числа экзо- и эндогенных патогенных факторов. Гигиенические исследования, проведенные в промышленно-развитых регионах Российской Федерации и ряде зарубежных стран, подтверждают зависимость формирования ХГД от концентрации экопатогенов, загрязняющих воздух, почву и воду [12-15]. В условиях максимально допустимого уровня токсических отходов промышленного производства продолжают проживать 73\% населения России.

Пристального внимания заслуживают дети, живущие в особых климатогеографических условиях. Одним из таких внешних факторов, оказывающих постоянное воздействие на все живые организмы, является аномальное геомагнитное поле. Железногорск - крупный населенный пункт, расположенный на территории Курской магнитной аномалии (КМА). Дети, проживающие в Железногорске, находятся под постоянным воздействием аномального геомагнитного поля, напряженность которого в 4-5 раз превышает фоновые значения, зарегистрированные в Курске. Однако в литературе отсутствуют данные о влиянии магнитных полей повышенной напряженности на формирование гастродуоденальной системы и ее патологии у детей.

Цель исследования: оценить особенности возникновения и течения заболеваний, формирования сопутствующей патологии, а также состояние ферментных систем желудочно-кишечного тракта у детей с ХГД, проживающих на территории КМА и подвергающихся постоянному воздействию геомагнитного поля повышенной напряженности.

\section{ПАЦИЕНТЫ И МЕТОДЫ}

\section{Участники исследования}

В 2008-2009 гг. под нашим наблюдением находились 120 детей в возрасте от 5 до 15 лет (60 детей из г. Курска и 60 - из г. Железногорска) с верифицированным диагнозом "Хронический гастродуоденит". Пациентов отбирали методом случайной выборки. Статистически значимых различий в возрастном и гендерном составе обеих групп не установлено. Был тщательно собран анамнез жизни и болезни. При анализе результатов учитывали длительность заболевания и наличие наследственной отягощенности по данному виду патологии.

\section{Методы исследования}

Всем детям проведено комплексное клинико-лабораторное и инструментальное обследование, включавшее клинический анализ крови и мочи, биохимический анализ крови, исследование крови на содержание глюкозы, микроскопическое исследование кала (копрограмма), ультразвуковое исследование печени, желчного пузыря, поджелудочной железы.

\section{Статистическая обработка данных}

Статистический анализ фактических данных осуществляли при помощи пакета программ Statistica v. 6.0. Результаты проанализированы при помощи методов описательной статистики с определением средней арифметической (М) и среднего квадратичного отклонения $(\sigma)$. Оценка достоверности различий для данных, имеющих нормальное распределение, проводилась с использованием $t$-критерия Стьюдента. Достоверность различий в копрограммах детей, представленных в виде процентных долей, рассчитывали с применением углового преобразования Фишера (F). Различия считали статистически значимыми при $p \leqslant 0,05$.

\section{РЕЗУЛЬТАТЫ}

В течение долгого периода времени обращало на себя внимание широкое распространение болезней органов пищеварения среди детского населения г. Железногорска. Заболеваемость гастритами и гастродуоденитами в Железногорске в 2,7 раза превышала показатели их встречаемости в Курске. С 2005 г. обнаружилась тенденция к снижению уровня заболеваемости гастритами и гастродуоденитами в Железногорске, что связано с увеличением охвата диспансеризацией детского населения и активным внедрением новых методов лечения и профилактики. Так, заболеваемость гастродуоденитами в Железногорске в 2004 г. составила 113,2, а в 2009 г. - 88,3 на 100 тыс. детей, тогда как в Курске 41,7 и 34,7 в 2004 и в 2009 гг., соответственно (рис. 1).

При оценке длительности заболевания установлено, что 50\% детей Железногорска имеют стаж болезни от 2 до 7 лет, у 40\% пациентов ХГД выявлен менее 1 года назад, а 10\% детей страдают им более 7 лет. В Курске распределение заболеваний по их длительности носило иной характер: у 35\% детей ХГД был диагностирован в течение последнего года, у 35\% длительность болезни составила от 2 до 7 лет, и у 30\% пациентов диагноз был верифицирован более 7 лет назад. При тщательном сборе анамнеза обращала на себя внимание ранняя манифестация ХГД у детей в Железногорске. Средний возраст дебюта заболевания в Курске был равен 6,4 \pm 0,4, в Железногорске - 5,0 \pm 0,4 года ( $p \leqslant 0,01)$.

У всех детей в обеих группах зарегистрирована сопутствующая патология, связанная как с дополнительным поражением желудочно-кишечного тракта, так и с вовлечением других органов и систем. Структура сопутствующей патологии у детей с ХГД представлена на рис. 2. 
Рис. 1. Заболеваемость хроническим гастродуоденитом у детей в Курске и Железногорске

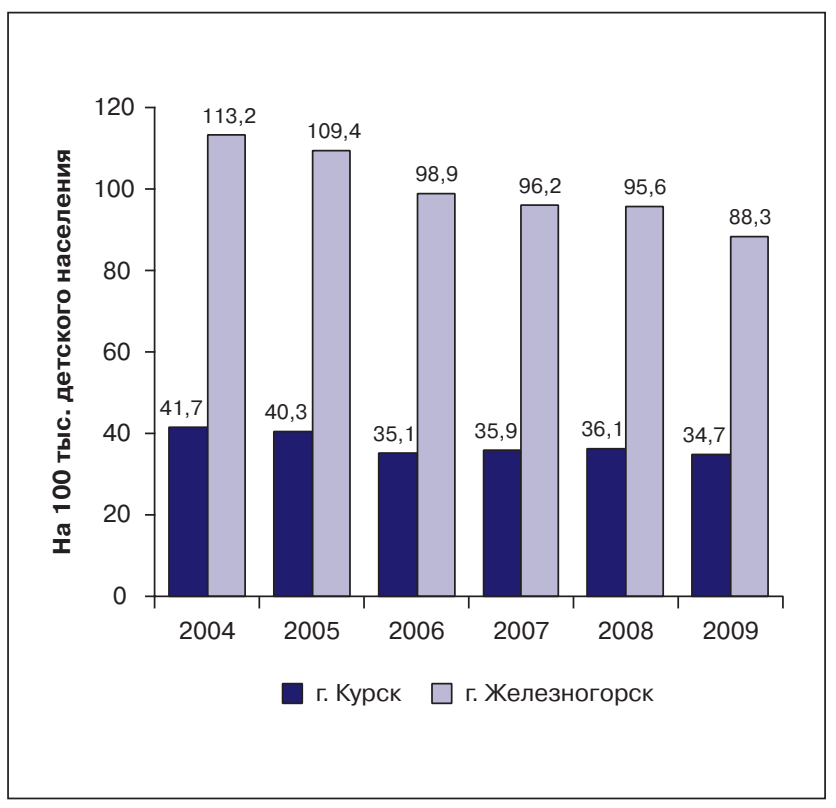

Рис. 3. Содержание жирных кислот в копрограмме у детей с хроническим гастродуоденитом в Курске и Железногорске

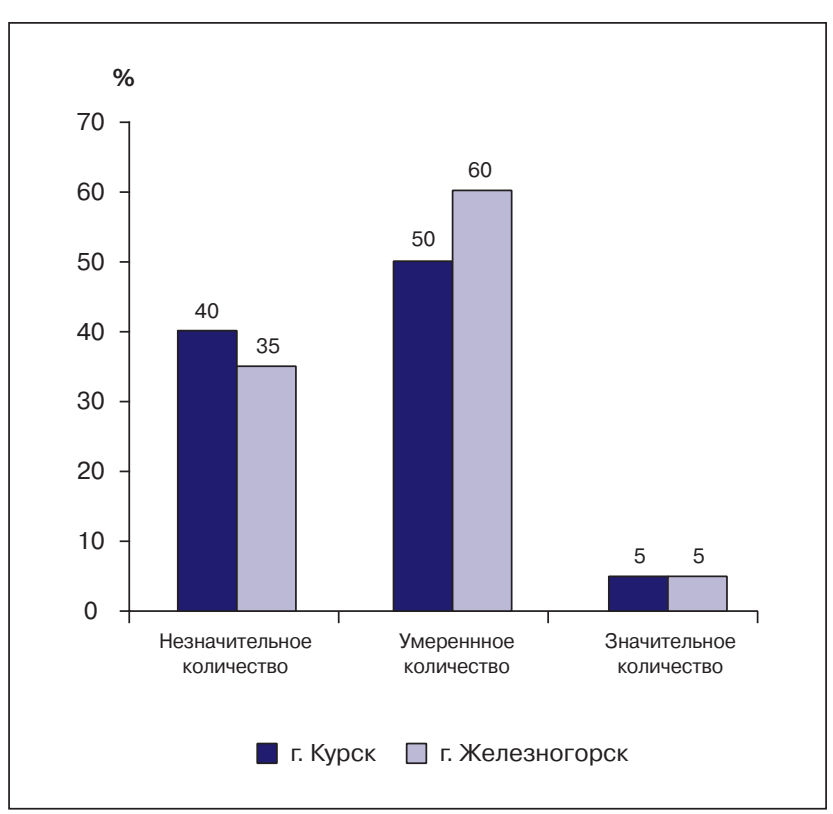

Анализ сопутствующей патологии показал, что в обеих группах преобладали реактивные изменения панкреатогепатобилиарной зоны, диагностированные по данным ультразвукового исследования. Эти изменения встречались у 27 (45\%) жителей Курска и 39 (65\%) человек из Железногорска. Врожденная аномалия в виде перегиба желчного пузыря регистрировалась с одинаковой частотой у детей обоих населенных пунктов. Нарушения динамики и реологии желчи, проявляющиеся в виде дисхолий и дискинезий желчевыводящих путей, чаще встречались у курских ( $n=21,35 \%)$, чем у железногорских детей ( $n=12,20 \%)$. Следует отметить большую распространенность аллергической патологии у детей, проживаю-
Рис. 2. Сравнительная характеристика сопутствующей патологии у детей с хроническим гастродуоденитом в Курске и Железногорске

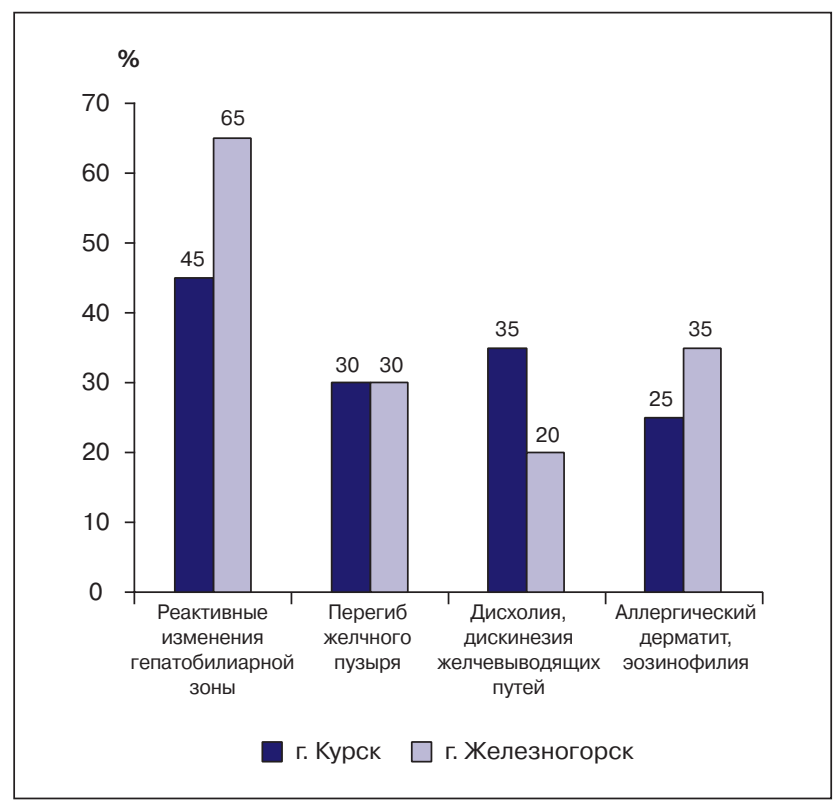

Рис. 4. Содержание солей жирных кислот в копрограмме у детей с хроническим гастродуоденитом в Курске и Железногорске

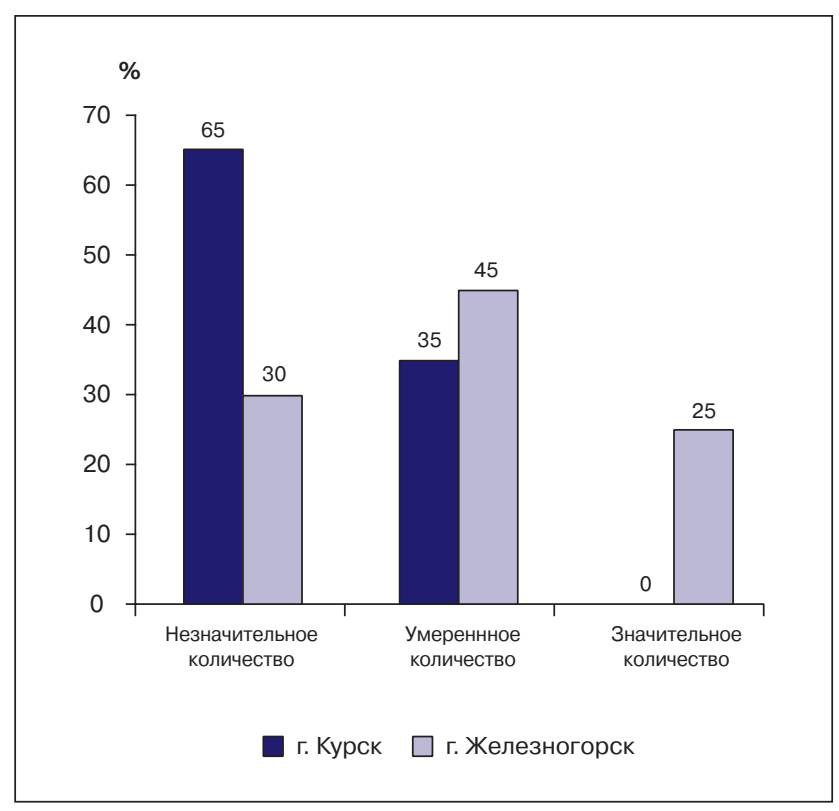

щих в аномальном геомагнитном регионе. Атопический дерматит в сочетании с ХГД диагностирован у 15 (25\%) пациентов из Курска и у 21 (35\%) из Железногорска. у 9 (15\%) человек из Железногорска ХГД сочетался с атопической бронхиальной астмой. У 12 пациентов из Железногорска верифицировано распространенное поражение желудочно-кишечного тракта (пангастрит, эрозивный бульбит).

Копрологическое исследование является вполне современным методом подтверждения функциональной недостаточности поджелудочной железы, желудка и кишечника, в связи с чем было целесообразно его проведение всем детям, участвовавшим в исследовании (рис. 3). 
У большинства обследуемых из обоих регионов при микроскопическом исследовании кала имело место умеренное количество (++) жирных кислот: у 50\% детей из региона с фоновыми значениями геомагнитного поля и у 60\%, проживающих в районе КМА. Однако незначительное количество жирных кислот (+) в копрограмме встречалось у $40 \%$ жителей Курска и у 35\% - Железногорска. Наличие в кале незначительного и умеренного количества жирных кислот может свидетельствовать о нарушении тонкокишечного переваривания и снижении липолитической активности желчи (рис. 4).

Умеренное повышение содержания мыл (солей жирных кислот) характерно для нарушения желудочного пищеварения, а также может встречаться у детей первого полугодия жизни, находящихся на грудном вскармливании. У большинства обследуемых из Курска соли жирных кислот в кале встречались в незначительном количестве (65\%), в Железногорске это явление было характерно для 30\% пациентов ( $p \leqslant 0,01)$. У $25 \%$ железногорских детей зафиксировали значительное количество солей жирных кислот в каловых массах, в то время как у курских пациентов подобные изменения не регистрировались $(p \leqslant 0,01)$.

В кале у детей с ХГД г. Курска чаще встречалось незначительное количество (+) крахмала (у 80\%), тогда как у 60\% пациентов Железногорска обнаружено его умеренное содержание $(++)$. Присутствие незначительного количества крахмала в норме допустимо у детей старших возрастных групп. Значительное количество $(+++)$ крахмала в каловых массах у детей, характерное для синдрома расстройства ротового пищеварения, обнаружено не было.

\section{REFERENCES}

1. Baranov A. A. Nauchnye i Organizatsionnye Prioritety v Detskoy Gastroenterologii [Scientific and Organizational Priorities in Pediatric Gastroenterology]. Pediatriya [Pediatrics]. 2002; 3: 12-18.

2. Denisov M.Yu. Prakticheskaya Gastroenterologiya dlya Pediatra [Practical Gastroenterology for Pediatricians]. Moscow, 1999. 295 p.

3. Korsunskiy A.A. Helicobacter pylori-assotsiirovannye Zabolevaniya kak Pediatricheskaya Problema [Helicobacter pylori-associated diseases as a pediatric problem]. Rossiyskiy Pediatricheskiy Zhurnal [Russian Journal of Pediatrics]. 2002; 5: 48-50.

4. Mazurin A.V., Tsvetkova L. N., Filin V.A. Aktual'nye Voprosy Detskoy Gastroenterologii [Topical issues of Pediatric Gastroenterology]. Pediatriya [Pediatrics]. 2000; 5: 19-22.

5. Baranov A.A. Sostoyanie Zdorov'ya Detey i Podrostkov v Sovremennykh Usloviyakh. Problemy i Puti Resheniya [Health of Children and Adolescents under Present Conditions. Problems and Solutions]. Rossiyskiy Pediatricheskiy Zhurnal [Russian Journal of Pediatrics]. 1998; 1: 5-8.

6. Volkov A.I., Usanova E.P. Dinamika Epidemiologicheskikh Pokazateley Zabolevaemosti Organov Pishchevareniya u Detey. Materialy VII Kongressa Pediatrov Rossii [Dynamics of Epidemiological Incidence Rates of Digestive Organs in Children. Proceedings of the VII Congress of Pediatricians of Russia]. Moscow, 2002. P. 54-55.

7. Baranov A.A. Ekologicheskie i Gigienicheskie Problemy Pediatrii [Environmental and Hygienic Problems in Pediatrics]. Rossiyskiy Pediatricheskiy Zhurnal [Russian Journal of Pediatrics] 1999; 3: 5-7.

8. Volkov A.I. Khronicheskiy Gastroduodenit i Yazvennaya Bolezn' $u$ Detey [Chronic Gastroduodenitis and Peptic Ulcer in Children]. RM [Russian Journal of Medicine]. 1999; 4: 179-186.

9. Pechkurov D. V., Shcherbakov P.L., Kachanova T. I. Ispol'zovanie Klinicheskikh Testov v Opredelenii Prioritetnosti Obsledovaniya s

\section{ЗАКЛЮЧЕНИЕ}

Хронические заболевания желудка и двенадцатиперстной кишки регистрируют у детей в регионе Курской магнитной аномалии (Железногорск) в 2,7 раза чаще, чем в Курске. Среди железногорских пациентов отмечен достоверно более ранний дебют заболевания (5,0 \pm 0,4 года), чем в Курске (6,4 \pm 0,4 года; $p \leqslant 0,01)$. В качестве сопутствующей патологии у детей Железногорска чаще встречаются реактивные изменения гепатобилиарной зоны и сочетание ХГД с аллергической патологией (аллергический дерматит, эозинофилия, бронхиальная астма). При изучении копрограмм у большинства пациентов из Железногорска выявлены изменения в виде наличия умеренного $(++)$ и значительного $(+++)$ количества жирных кислот и умеренного количества крахмала (++). У детей г. Курска, страдающих ХГД, жирные кислоты, мыла и крахмал присутствовали в кале в незначительном (+) количестве. Указанные изменения, более выраженные у пациентов, находящихся под постоянным воздействием геомагнитного поля повышенной напряженности, могут свидетельствовать о существенных нарушениях процессов тонкокишечного переваривания и недостаточности ферментативной активности отдельных компонентов пищеварительных соков. Таким образом, детей с ХГД, проживающих на территории Курской магнитной аномалии, следует выделить в особую группу пациентов. Это будет служить основанием для рациональной коррекции стандартных схем лечения хронических заболеваний желудка и двенадцатиперстной кишки у детей с учетом особенностей их течения в данном регионе.

Sindromom Dispepsii [Using Clinical Tests in Prioritizing Dyspepsia Syndrome Examination]. Pediatriya [Pediatrics]. 2004; 2: 4-8. 10. Pechkurov D. V. Epidemiologiya Gastroenterologicheskikh Zabolevaniy u Detey: Dostovernost' Retrospektivnogo Analiza [Epidemiology of Gastrointestinal Diseases in Children: The Accuracy of Retrospective Analysis]. Pediatriya [Pediatrics]. 2004; 3: 22-23. 11. Parmenova L.P. Endogennaya Intoksikatsiya u Detey $s$ Zabolevaniyami Vnutrennikh Organov Pishchevaritel'nogo Trakta [Endogenous Intoxication in Children with Diseases of the Digestive Tract Internal Organs]. Pediatriya [Pediatrics]. 2004; 2: 15-19.

12. Anfinogenova O.B., Davydov B.I. Diagnosticheskiy Algoritm Khronicheskogo Gastroduodenita Promyshlennogo Regiona Zapadnoy Sibiri - Kuzbassa [Diagnostic Algorithm of Chronic Gastroduodenitis in the Industrial Region of Western Siberia Kuzbass]. Sibirskiy Meditsinskiy Zhurnal [Siberian Journal of Medicine]. 2004; 19 (2): 82-83.

13. Zvyagina E.V. Khronicheskie Zabolevaniya Verkhnego Otdela Pishchevaritel'nogo Trakta u Detey Kemerova [Chronic Diseases of the Upper Digestive Tract in Children from Kemerovo]. Avtoreferatdissertatsiya kandidata meditsinskikh nauk [Autoabstract-thesis of Candidate of Medical Sciences]. Tomsk, 2000. 24 p.

14. Davydov B.I., Anfinogenova O.B., Zvyagina E.V. Kliniko-Instrumental'naya Diagnostika Khronicheskikh Gastroduo-Denitov u Detey g. Kemerovo [Clinical and Instrumental Diagnosis of Chronic Gastroduodenitis in Children from Kemerovo]. Sibirskiy Meditsinskiy Zhurnal [Siberian Journal of Medicine]. 2003; 18 (4): 19.

15. Shcheplyagina L.A., Ursova N.I., Abramova I.Yu. Rol' Faktorov Ekologicheskogo Riska v Razvitii Zabolevaniy Organov Pishchevareniya u Detey po Materialam Moskovskoy Oblasti [Role of Environmental Risk Factors in the Development of Digestive Diseases in Children Based on Materials from the Moscow Region]. Rossiyskiy zhurnal gastroenterologii, gepatologii, koloproktologii [Russian Journal of Gastroenterology, Hepatology and Coloproctology]. 2000; 5 (Append. 11): 109. 